\title{
Heterogeneity of treatment effects in trials on psychotherapy of depression
}

Tim Kaiser ${ }^{1}$, Constantin Volkmann ${ }^{2}$, Alexander Volkmann ${ }^{3}$, Eirini Karyotaki ${ }^{4}$, Pim Cuijpers $^{4}$, Eva-Lotta Brakemeier ${ }^{1}$

1: Department of Clinical Psychology and Psychotherapy, University of Greifswald, Germany

2: Department of Psychiatry and Psychotherapy, Charité - University Medical Campus Berlin, Germany

3: Independent Researcher, Berlin, Germany

4: Department of Clinical Psychology, Amsterdam, The Netherlands

\section{Corresponding author:}

Dr. Tim Kaiser

Department of Psychology

University of Greifswald

Franz-Mehring-Straße 47, 17489 Greifswald, Germany

Email: tim.kaiser@uni-greifswald.de

\begin{abstract}
Practitioners and researchers alike assume that there is individual variability in the effects of treatments for mental disorders. However, for psychotherapy, up to now this assumption has never been empirically tested. Using a large database of randomized-controlled trials on psychotherapy of depression in adults (306 trials including a total of 51.853 patients), we performed a Bayesian variance ratio meta-regression. For the entire sample, we found a $9 \%$ higher variance in the intervention groups compared to the control groups. Depending on the depression scale used, this corresponds to a standard deviation of the individual treatment effect of 3-4 points. Subgroup analyses revealed that the effect variability of some types of therapy is larger than others. Our results are the first to indicate that patients do benefit differently from psychotherapy. We conclude that there is a sound basis for the paradigm of personalized psychotherapy, which brings about implications for both research and clinical practice.
\end{abstract}

Public Health Significance Statement: In recent years, studies with high methodological quality have pointed out that the efficacy of psychotherapy in the treatment of depression is less satisfactory than previous research suggested. To optimize psychotherapy for nonresponders, the paradigm of personalized therapy is coming into the research focus. In this study, we show for the first time that the effects of psychological interventions vary more than those of control conditions. This shows that differential response to treatments is inherent to intervention effects. Thus, it could indeed be beneficial to better tailor psychotherapy to individual patients. In practice, session-by-session outcome monitoring should be used to detect non-responding cases in ongoing treatments. Statistical methods guiding the selection of treatment components capitalize on the heterogeneity of treatment effects and are thus likely to improve outcomes.

These findings pave the way for broad research and implementation of approaches that support personalization (e.g., monitoring and feedback systems) as well as a new shaping of training beyond the traditional schools of thought in psychotherapy.

Keywords: Meta-Analysis, Heterogeneity of Treatment Effects, Psychotherapy, Depression

(C) 2022, American Psychological Association. This paper is not the copy of record and may not exactly replicate the final, authoritative version of the article. Please do not copy or cite without authors\&apos; permission. The final article will be available, upon publication, via its DOI: 10.1037/cps0000079 


\section{Introduction}

The question of the effectiveness of psychotherapy has preoccupied researchers for decades (Smith \& Glass, 1977). Recent results of psychotherapy outcome studies for depression show robust but modest effects that leave room for improvement (Cuijpers et al., 2019). Only 41\% of depressed patients respond to psychotherapy (Cuijpers, Karyotaki, et al., 2021) compared to $17 \%$ in control groups. Additionally, $20 \%$ of patients discontinue their treatment prematurely (Cooper \& Conklin, 2015; Swift \& Greenberg, 2012). Although its long-term effects are superior to pharmacological treatment (Furukawa, Shinohara, et al., 2021), more than half of depressed patients treated with psychotherapy show a relapse a few years after treatment (Steinert et al., 2014). Taken together, these robust findings provide an incentive for various methodological optimization procedures that could improve outcomes.

Personalized medicine aims to determine which characteristics of an individual patient predict the outcome of a particular treatment to achieve a better match between the individual and the treatment received (Simon \& Perlis, 2010). This approach is based on the assumption that patients suffering from a certain disorder have different treatment effects. This assumption is not limited to somatic medicine but was part and parcel of clinical psychology since its beginnings as an empirical science. The mandate to study this assumption was first formulated by a founding father of modern clinical psychology, Gordon Paul. His famous question, "What treatment, by whom, is most effective for this individual with that specific problem, and under which set of circumstances, and how does it come about?" (Paul, 1967, p. 111) provides a compact summary of the numerous possible vectors of personalization that should be given special attention in psychotherapy research. Even earlier, research on the question of which patients are likely to respond to psychotherapy was initiated by Barron (1953). Over the decades, methods for predicting psychotherapy outcomes for individual patients became more and more methodologically refined and were tested on larger samples. Instead of predicting the response to only one treatment, newer methods attempt to predict the outcome of multiple treatment alternatives (e.g., DeRubeis et al., 2014). The purpose of these treatment selection methods is to select the intervention that leads to the greatest possible treatment success for the individual patient. Even in the absence of statistical prediction models for treatment outcomes, clinical psychologists and psychotherapists routinely tailor their treatments to the individual patient by selecting from a finite set of possible intervention to achieve the greatest possible success (Cohen $\&$ DeRubeis, 2018). Although meta-analytic evidence clearly shows that statistical prediction models are, on average, superior to intuitive clinical judgments (Ægisdóttir et al., 2006), research on treatment selection in psychotherapy is still inconclusive. A recent review of empirical studies on statistical treatment selection methods in psychotherapy (LorenzoLuaces et al., 2020) concludes that these different methods of personalization improve the outcomes somewhat, but many of these studies lack statistical power or do not validate their methods on hold-out samples, thus possibly overestimating the effects. Regardless of whether it is based on clinical intuition or statistical decision-making rules, it is still unclear how much the effect of psychotherapy can be increased by interventions tailored to the individual patient.

Formally, the personalization of psychotherapy by treatment selection can only be successful under the assumption that not every treatment has the same effect for every patient. Instead, certain patient characteristics cause variance in the effect of available treatments. This is reflected by the variance of the individual treatment effect, which the literature refers to as the heterogeneity of treatment effects (HTE; Kessler \& Luedtke, 2021; Luedtke \& Kessler, 2021). The assumption that not every patient shows the same treatment effect is by no means self-evident. In addition to the therapy-specific treatment effect, the overall response to treatment consists of several other components, such as regression to the mean, spontaneous remission, placebo effects, or common factors (Enck \& Zipfel, 2019). 
Randomized-controlled trials are needed to isolate the therapy-specific effect by comparing average post-treatment symptom scores of the active group to a control group. HTE can be studied by comparing variances of post-treatment scores instead of means. By dividing the post-treatment score variance of the active treatment group by that of the control group, a variance ratio (VR) is obtained. Because only the effect of treatment in the active treatment group can lead to a difference from the control group, higher variance in the active group (i.e., a VR larger than 1) suggests interindividual differences in the actual treatment effect. Meta-analyses on the VR are increasingly used to find evidence on HTE (Winkelbeiner et al., 2019).

It is important to note that treatments that show constant but small effects are possible, as are treatments that show no effect on average but work particularly well or poorly for certain subgroups. For example, Plöderl and Hengartner (2019) found no evidence for HTE in a database of 163 placebo-controlled trials. It was concluded that pharmacological treatments of depression do not show HTE, challenging the assumption that subgroups of patients more likely to respond to these treatments exist. Still, a recent meta-analysis found only a small average treatment effect for antidepressant medication (Cipriani et al., 2018).

A demonstration of the presence and extent of HTE in psychotherapy for depression should be of great interest to researchers developing tailored approaches in psychotherapy and practitioners who apply them. Thus, we conduct the first meta-analysis on HTE in psychotherapeutic interventions. In doing so, we intend to answer the following three questions:

1. Is there heterogeneity of treatment effects in psychotherapy for depression?

2. Do the various types of psychotherapy differ concerning HTE?

3. How large is the improvement that can be achieved through a possible personalization of psychotherapy?

\section{Data acquisition}

\section{Methods}

We used a meta-analytic database of randomized studies on the psychological treatment of depression in adults (Cuijpers, 2008). Means and standard deviations (SDs) of endpoint scores (post-treatment) were obtained directly from the included articles. Sufficient data from 306 studies including a total of 51853 subjects were available. A total of 43 studies used active control groups that were not included in the main analyses. The data set we used can be obtained from P. Cuijpers upon request.

\section{Data analyses}

We conducted a Bayesian random-effects meta-regression. Following the previous studies (Cortés et al., 2019; Plöderl \& Hengartner, 2019; Volkmann et al., 2020), we used the logarithmic variance ratio ( $\operatorname{lnVR}$ ) as an outcome measure and indicator for HTE.

An $\ln$ VR that deviates from 0 may be regarded as evidence of HTE. $\ln$ VR measures the overall effect of an intervention on outcome variability. If the direct effect of a treatment on outcome variability is to be estimated, lnVR may yield biased results as it does not correct for a possible mean-variance relationship (Nakagawa et al., 2015). A mean-variance relationship is likely to occur if floor effects in outcome are present. In this case, variances in groups with lower endpoint scores are underestimated, leading to an underestimation of HTE. Therefore, we modeled the influence of differences in mean scores by including the logarithm of the ratio of mean treatment group scores to mean control group scores (logarithmic endpoint ratio, lnER) as a covariate. As recommended by Moyaert et al. (2017), we handled studies reporting multiple outcomes by conducting a three-level meta-analysis that allowed for variation from outcome scale to outcome scale, as well as variation between studies. 
We repeated this analysis for seven different types of psychotherapy: behavioral activation therapy, cognitive-behavioral therapy, third-wave approaches, psychodynamic therapy, interpersonal therapy, problem-solving therapy, supportive therapy, and "others". The "others" category contains procedures of which there were no more than ten studies in the database (Cuijpers et al., 2020). These include life-review therapy, cognitive behavioral analysis system of psychotherapy (CBASP), spiritual approaches, and therapies not following any particular theoretical approach.

Bayesian statistics allow for the a priori specification of the expected results ("priors"). As this is the first meta-analysis on HTE in psychotherapy, we decided to make only weak assumptions and therefore used priors that contain little information. For the intercept and slope coefficients, a Cauchy distribution with a location parameter of 0 and a scale parameter of 1 was specified as prior. Neither mean nor variance is defined for Cauchy distributions, so this specification can be considered weakly informative. "Half-Cauchy" priors with a location parameter of 0 and a scale parameter of 1 were specified for the between-study variance. Half-Cauchy priors take only positive values, which is adequate, as variances can only be positive.

For all parameter estimates 95\% credible intervals (CrIs) were calculated. In Bayesian statistics, $95 \%$ CrIs indicate the range in which the true parameter value lies with a probability of $95 \%$ given the evidence. All statistical analyses were carried out using the R programming language ( Version 4.0.2; R Core Team, 2020). Bayesian statistical modeling was conducted using the brms package (Bürkner, 2017).

\section{Bounds on the heterogeneity of treatment effects}

Although a VR that differs from one implies a variation of the treatment effect, the translation of VR to HTE is not straightforward and requires further computational steps. Based on the results of our meta-regression, we determined the bounds of the treatment effect using the analytical approach proposed by Volkmann (A. Volkmann, 2020). This approach assumes that every patient has a "true" treatment effect score for both treatment and the control condition, of which only one can be observed. The following formula determines the standard deviation of the individual treatment effect by adding the variance implied by the VR to the standard deviation of control group endpoint scores:

$$
\left.S D_{t x}=S D_{p} *\left(\sqrt{\left(V R^{2}-1+\rho^{2}\right.}\right)-\rho\right)
$$

Here, $\mathrm{SD}_{\mathrm{tx}}$ is the standard deviation of individual treatment effects, $\mathrm{SD}_{\mathrm{p}}$ is the standard deviation of post-treatment scores in the control condition, and VR is the estimate of the observed variance ratio. Finally, $\rho$ denotes the correlation between the treatment effect and the endpoint score under the control condition. For example, $\rho=-0.5$ would indicate that a patient who has low symptom scores after receiving the active treatment would have shown above-average symptom scores in the control condition. The lower $\rho$ is, the larger the HTE for a given VR. Conversely, $\rho=0.5$ would indicate that patients with a better outcome under the control treatment would also show a stronger treatment effect and HTE would be relatively low for a given VR. Since an individual patient can only receive either the active treatment or the control intervention in a given clinical trial, this correlation is not observable. Therefore, we will calculate the bounds of HTE for a range of eleven values of $\rho$, ranging from -1.0 to 1.0 by steps of 0.2 . Furthermore, by entering the upper and lower values of the $95 \% \mathrm{CrI}$ of the VR, the equation allows us to obtain $95 \%$ CrIs for the standard deviation of the treatment effect for every assumed value of $\rho$. This way, the theoretical bounds of HTE in psychotherapy for depression can be determined. 
Estimating the likelihood to observe differential response. To further contextualize our results, we conducted a simulation analysis of the probability of observing patients who will show meaningfully different outcomes to different treatments. Based on our results, we simulated possible endpoint scores and tested the probability of observing clinically relevant differences between hypothetical treatments. We simulated endpoint data sets by sampling from a normal distribution with the average observed endpoint scores as means and the computed individual standard deviations for the entire range of $\rho$ values. Simulations were conducted for four common outcome measures that are frequently used in clinical trials as well as in routine practice. The Beck Depression Inventory-II (BDI-II; Beck et al., 1996) is a 21-item self-report measure of depressive symptoms. The items are scored on a scale ranging from 0 to 3 . The total sum score of items indicates the severity of the depressive disorder. Psychometric evidence suggests that the BDI-II shows excellent reliability and high criterionbased validity (Wang \& Gorenstein, 2013). The Patient Health Questionnaire-9 (PHQ-9; Kroenke \& Spitzer, 2002) is a brief measure designed to assess the DSM-IV criteria of major depressive disorder. On nine items scored on a scale from 0 ("not at all") to 3 ("nearly every day"), the occurrence of depressive symptoms is measured. The PHQ-9 shows good reliability and criterion validity (Levis et al., 2019).

In addition to these self-report measures, two clinician-rated scales were included in this analysis. The Hamilton Depression Rating Scale (Hamilton, 1960) is a severity measure of depressive symptoms. The most common version of the HDRS consists of 21 items that are rated on scales ranging either from 0 to 4 or from 0 to 2 . It is frequently used in clinical studies of depression treatments. A recent review concluded that the HDRS possesses good interrater and test-retest reliability and can discriminate between various levels of symptom severity (Carrozzino et al., 2020). Finally, the Montgomery-Åsberg Depression Rating Scale (Montgomery \& Åsberg, 1979) is a ten-item rating scale designed to achieve maximum sensitivity to change. The MADRS shows high reliability and can discriminate between different levels of severity (Carmody et al., 2006).

For each scale and value of $\rho, 1000$ data sets with endpoint scores of 100 patients were simulated. We formed all 4950 possible combinations of two and calculated difference values for these data sets. These difference values were then compared to minimal clinically important difference (MCID) scores reported in the literature. For each simulated data set, we counted the number of difference scores that exceed the MCID. The average proportion of difference scores exceeding the MCID for all 1000 data sets per $\rho$ value and outcome measure indicates the likelihood of observing clinically different outcomes. For example, if $35 \%$ of difference scores exceed the MCID, the likelihood that a randomly selected patient will have a clinically meaningful benefit is $35 \%$.

We assumed the following minimal clinically important differences (MCID) reported in the literature: five points on the BDI-II (Hiroe et al., 2005), five points on the PHQ-9 (Löwe et al., 2004), five points on the HDRS (Bobo et al., 2016), and eight points on the MADRS (Leucht et al., 2017).

\section{Results}

Table 1 summarizes the results of all models, including subgroup analyses. For the whole sample, the posterior mean estimate for the intercept $(\mu)$ was 0.09 (95\% CrI: $0.06 ; 0.14)$. The slope coefficient for $\operatorname{lnER}$ was 0.50 ( $\beta_{\operatorname{lnER}}, 95 \% \mathrm{CrI}$ : $\left.0.42 ; 0.59\right)$, showing a clear meanvariance relationship in the data. There was substantial between-study variance, with a standard deviation of the intercept $(\tau)$ estimated at 0.13 (95\% CrI: $0.11 ; 0.16)$. The variance between outcome measures was low, with an estimate of 0.02 (95\% CrI: 0.00; 0.06), suggesting that the observed results are unlikely to be explained by the choice of different outcome scales. 
The subgroup analyses revealed that VRs differed between treatments. The maximum estimate was observed for behavioral activation therapy with $\mu=.28$ (95\% CrI: $0.02 ; 0.54)$. Conversely, interpersonal therapy and problem-solving therapy showed VRs compatible with a near-constant treatment effect. However, credible intervals for all VR estimates overlapped, so there was limited evidence for clear differences between treatment types.

Table 1. Results of meta-regression models, including subgroup analyses by treatment type.

\begin{tabular}{|c|c|c|c|c|c|c|}
\hline & Trials & $\begin{array}{l}\text { Subjects } \\
\text { Treatment }\end{array}$ & Control & $\begin{array}{l}\text { lnVR Meta-regression } \\
\mu \text { (Intercept), } 95 \% \mathrm{CrI}\end{array}$ & $\beta_{\operatorname{lnER}, 95 \% \mathrm{CrI}}$ & $\tau, 95 \% \mathrm{CrI}$ \\
\hline All types & 306 & 27785 & 24068 & $\begin{array}{l}0.09 \\
{[0.06 ; 0.14]}\end{array}$ & $\begin{array}{l}0.48 \\
{[0.38 ; 0.58]}\end{array}$ & $\begin{array}{l}0.13 \\
{[0.11 ; 0.16]}\end{array}$ \\
\hline BAT & 22 & 1602 & 1394 & $\begin{array}{l}0.28 \\
{[0.02 ; 0.54]}\end{array}$ & $\begin{array}{l}0.88 \\
{[0.31 ; 1.49]}\end{array}$ & $\begin{array}{l}0.13 \\
{[0.01 ; 0.29]}\end{array}$ \\
\hline CBT & 181 & 14645 & 12895 & $\begin{array}{l}0.09 \\
{[0.04 ; 0.14]}\end{array}$ & $\begin{array}{l}0.49 \\
{[0.37 ; 0.61]}\end{array}$ & $\begin{array}{l}0.13 \\
{[0.09 ; 0.16]}\end{array}$ \\
\hline PDT & 9 & 952 & 904 & $\begin{array}{l}0.05 \\
{[-0.17 ; 0.29]}\end{array}$ & $\begin{array}{l}0.14 \\
{[-0.73 ; 1.04]}\end{array}$ & $\begin{array}{l}0.14 \\
{[0.01 ; 0.39]}\end{array}$ \\
\hline IPT & 21 & 1603 & 1463 & $\begin{array}{l}-0.04 \\
{[-0.18 ; 0.11]}\end{array}$ & $\begin{array}{l}0.17 \\
{[-0.21 ; 0.5]}\end{array}$ & $\begin{array}{l}0.09 \\
{[0.01 ; 0.20]}\end{array}$ \\
\hline PST & 20 & 2077 & 1877 & $\begin{array}{l}0.00 \\
{[-0.09 ; 0.09]}\end{array}$ & $\begin{array}{l}0.21 \\
{[-0.01 ; 0.45]}\end{array}$ & $\begin{array}{l}0.05 \\
{[0.00 ; 0.15]}\end{array}$ \\
\hline ST & 15 & 1106 & 527 & $\begin{array}{l}0.10 \\
{[-0.12 ; 0.35]}\end{array}$ & $\begin{array}{l}0.60 \\
{[-0.26 ; 1.56]}\end{array}$ & $\begin{array}{l}0.07 \\
{[0.00 ; 0.22]}\end{array}$ \\
\hline $\begin{array}{l}\text { Third- } \\
\text { wave }\end{array}$ & 22 & 1222 & 988 & $\begin{array}{l}0.15 \\
{[-0.02 ; 0.33]}\end{array}$ & $\begin{array}{l}0.68 \\
{[0.26 ; 1.15]}\end{array}$ & $\begin{array}{l}0.06 \\
{[0.00 ; 0.18]}\end{array}$ \\
\hline Other & 48 & 4454 & 3958 & $\begin{array}{l}0.14 \\
{[0.02 ; 0.25]}\end{array}$ & $\begin{array}{l}0.18 \\
{[0.06 ; 0.78]}\end{array}$ & $\begin{array}{l}0.14 \\
{[0.06 ; 0.20]}\end{array}$ \\
\hline
\end{tabular}

Note. 95\% CrI: credible interval. BAT: behavioral activation therapy, CBT: cognitivebehavioral therapy, PDT: psychodynamic therapy, IPT: interpersonal psychotherapy, PST: problem-solving therapy, ST: supportive therapy, Third-wave: third-wave cognitivebehavioral approaches, Other: miscellaneous approaches. $\mu$ : Intercept, $\beta_{\operatorname{lnER}}$ : slope coefficient of the logarithmic endpoint ratio, $\tau^{2}$ : between-study heterogeneity. Some trials included more than one type of psychotherapy, so the total number of trials of all subgroups is larger than the number of trials reported under "all types".

Using the point estimate of the variance ratio $\left(\mathrm{e}^{\mu}=1.09\right)$ and the weighted average standard deviations of control group endpoint scores, we proceeded to determine the extent and bounds of HTE. The following equations were used:

$$
\begin{gathered}
S D_{\text {tx BDI }}=10.02 *\left(\left(\sqrt{0.20+\rho^{2}}\right)-\rho\right) \\
S D_{\text {tx } P H Q-9}=5.81 *\left(\left(\sqrt{0.20+\rho^{2}}\right)-\rho\right) \\
S D_{\text {tx HDRS }}=6.60 *\left(\left(\sqrt{0.20+\rho^{2}}\right)-\rho\right) \\
S D_{\text {txMADRS }}=9.22 *\left(\left(\sqrt{0.20+\rho^{2}}\right)-\rho\right)
\end{gathered}
$$

Table 2 summarizes the estimates of standard deviations of the treatment effect. Additionally, the average probabilities of patients showing a clinically relevant treatment effect assuming this standard deviation are shown. In figure 1, the upper and lower bounds of HTE are depicted and compared to observable HTE if the variance ratio were exactly one. 
Table 2. Estimates of the standard deviation of individual treatment effects and the likelihood of differential effects for four outcome measures.

\begin{tabular}{|c|c|c|c|c|c|c|c|c|}
\hline \multirow[b]{2}{*}{$\rho$} & \multicolumn{2}{|l|}{ BDI-II } & \multicolumn{2}{|l|}{ PHQ-9 } & \multicolumn{2}{|l|}{ HDRS } & \multicolumn{2}{|c|}{ MADRS } \\
\hline & $\mathrm{SD}_{\mathrm{Tx}}$ & $\begin{array}{c}\delta_{\text {BDI-II }}> \\
5\end{array}$ & $\mathrm{SD}_{\mathrm{Tx}}$ & $\begin{array}{c}\delta_{\mathrm{PHQ}-9} \\
>5\end{array}$ & $\mathrm{SD}_{\mathrm{Tx}}$ & $\begin{array}{c}\delta_{\mathrm{HDRS}} \\
>5\end{array}$ & $\mathrm{SD}_{\mathrm{Tx}}$ & $\begin{array}{c}\delta_{\text {MADRS }}> \\
8\end{array}$ \\
\hline-1.0 & 21.02 & $79 \%$ & 12.19 & $68 \%$ & 13.84 & $73 \%$ & 19.33 & $69 \%$ \\
\hline-0.8 & 17.23 & $79 \%$ & 9.99 & $65 \%$ & 11.34 & $71 \%$ & 15.84 & $67 \%$ \\
\hline-0.6 & 13.54 & $77 \%$ & 7.85 & $61 \%$ & 8.92 & $67 \%$ & 12.45 & $62 \%$ \\
\hline-0.4 & 10.06 & $72 \%$ & 5.83 & $52 \%$ & 6.62 & $59 \%$ & 9.25 & $53 \%$ \\
\hline-0.2 & 6.96 & $61 \%$ & 4.4 & $37 \%$ & 4.58 & $45 \%$ & 6.40 & $38 \%$ \\
\hline 0.0 & 4.53 & $44 \%$ & 2.63 & $18 \%$ & 2.98 & $25 \%$ & 4.17 & $17 \%$ \\
\hline 0.2 & 2.95 & $23 \%$ & 1.71 & $4 \%$ & 1.94 & $7 \%$ & 2.71 & $4 \%$ \\
\hline 0.4 & 2.04 & $8 \%$ & 1.18 & $0 \%$ & 1.34 & $1 \%$ & 1.88 & $0 \%$ \\
\hline 0.6 & 1.52 & $2 \%$ & 0.88 & $0 \%$ & 1.00 & $0 \%$ & 1.39 & $0 \%$ \\
\hline 0.8 & 1.19 & $0 \%$ & 0.69 & $0 \%$ & 0.78 & $0 \%$ & 1.10 & $0 \%$ \\
\hline 1.0 & 0.98 & $0 \%$ & 0.57 & $0 \%$ & 0.64 & $0 \%$ & 0.90 & $0 \%$ \\
\hline $\mathrm{M}_{\mathrm{Tx}}$ & 14.74 & & 7.92 & & 10.85 & & 14.18 & \\
\hline
\end{tabular}

Note. The coefficient ' $\rho$ ' refers to the intraindividual correlation of treatment effect and the endpoint score under placebo. $\mathrm{SD}_{\mathrm{Tx}}$ : standard deviation of individual treatment effect. $\delta_{\mathrm{BDI}-\mathrm{II}}$ $>5$, etc.: the proportion of patients with a clinically relevant treatment effect. $\mathrm{M}_{\mathrm{Tx}}$ : samplesize weighted average of endpoint scores in treatment groups, added to provide context. 
Figure 1. Upper and lower bounds on the standard deviation of the treatment effect.

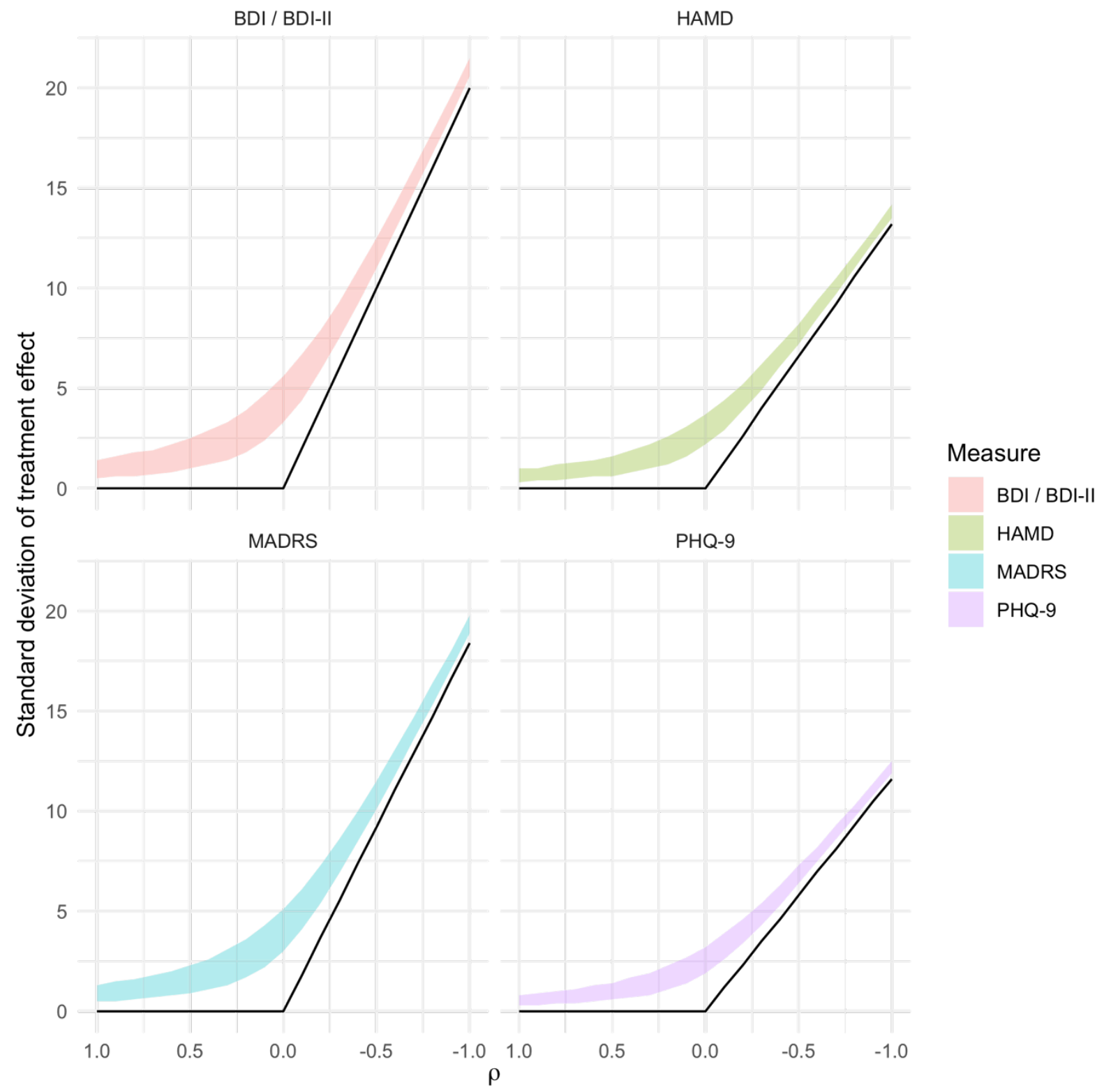

Note. Ranges are plotted for four commonly used outcome scales. They are based on the 95\% CrI of the VR estimate $(0.05 ; 0.14)$. The black line shows the standard deviation if the variance ratio between treatment and control conditions were 1 . 


\section{Discussion}

In this study, we were able to show for the first time that the variance ratio in psychotherapy outcomes is compatible with patient-by-treatment interaction, thus implying the existence of subgroups that respond especially well to various forms of psychotherapy. A simulation study based on these results allowed us to determine the probability of a patient responding meaningfully to different treatments. In the second step, we have translated the variance ratio into HTE that can be expressed in points on symptom scales. Finally, a simulation study provided an estimate for the chance of observing clinically meaningful differences under different treatments. Methods of personalizing psychotherapy that select the most effective treatment for an individual patient have so far implicitly assumed this variance component. In this article, we show for the first time that HTE indeed exists in psychotherapy for depression. Thus, treatment selection is likely to improve the effects of psychotherapy. As will be discussed below, this finding has important implications for both future studies on treatment selection methods and clinical practice.

Until now, the potential benefit of treatment selection methods had to be estimated from empirical studies suffering from small sample sizes and other methodological limitations (Lorenzo-Luaces et al., 2020). Thus, it was unclear how much such methods can be expected to contribute to treatment outcomes. Conducting clinical studies on treatment selection algorithms is costly and, without proper sample size planning and cross-validation, provides unreliable effect size estimates. Determining meaningful bounds on heterogeneity from existing clinical trial data offers the decided advantage that future studies can refer to these bounds. Thus, future studies do not run the risk of being underpowered and, at the same time, can determine in advance the maximum contribution that the proposed algorithms can make to the care situation of people suffering from depression.

The meta-analytically derived VR translates to a standard deviation of the treatment effect of 2.6 points on the PHQ-9 to 4.4 points on the BDI-II. This standard deviation can be considered as a benchmark for the effects that may be expected from statistical treatment selection methods in psychotherapy. For example, a realistic goal for the development of a treatment selection algorithm could be that it manages to increase the individual treatment effect of patients by one standard deviation of the individual treatment effect. Studies testing the efficacy of this algorithm should be planned so that the statistical power is sufficient to detect a difference of 3-4 points. A second important implication for future research is the potential effect of switching therapy approaches in the case of treatment non-response. Because patients can be expected to show different responses to different types of psychotherapy, switching approaches can be expected to bring about therapeutic change. Interestingly, subgroup analyses revealed that HTE possibly varies by psychotherapy approach. The heterogeneity for CBT, which closely follows the overall heterogeneity at $9 \%$, is the most robust as it is based on the largest dataset (181 studies). Other types of treatment, like BAT $(\operatorname{lnVR}=0.28)$ or third-wave approaches $(\operatorname{lnVR}=0.15)$ might have a larger HTE. For these therapy approaches, it appears particularly important to identify patients who respond particularly well. Given the number of possible mechanisms common to all psychotherapy methods (common factors), as well as specific factors (Wampold, 2015), there are several possible explanations for individual differences in outcomes. For example, BAT specifically addresses an assumed cause of depression - the loss of positive reinforcements due to avoidance behavior - and uses specific techniques to help patients increase the rate of value-based behavior to promote access to long-term positive reinforcements. It could be that patients with pronounced avoidance behavior particularly benefit here, while other patients derive less benefit from this method. In contrast, according to our analyses, therapies such as PDT, IPT, and PST seem to work (or not to work) equally well for all patients, as we find no evidence for HTE here. Generally, it can be concluded that therapies such as BAT, third wave approaches, as well as CBT can be further optimized by identifying patients who 
respond particularly well to these therapeutic approaches and to whom these therapies should then be specifically recommended. Our results also indicate that HTE is largest when responses are strongly and negatively correlated under treatment and control conditions. As Volkmann (2020, p. 3) points out, assumptions about this correlation can be informed by domain knowledge. For example, important implications for certain subtypes of depression that are unlikely to show spontaneous remission can be derived. Although on average, patients with persistent depressive disorder respond to psychotherapy and other treatments (Kriston et al., 2014), it was shown in longitudinal studies that these patients show chronically high symptom severity when not treated (Stegenga et al., 2012). This could imply that chronically depressed patients show a somewhat higher HTE because their treatment and control scores can be assumed to be negatively correlated. Further research on selecting the optimal treatment for this subtype of depression could be especially worthwhile. The wellestablished heterogeneity of depression (Goldberg, 2011; Price et al., 2019) might also have contributed to HTE because it is likely that most of the included studies included patients with different subtypes of depression. HTE in other less heterogeneous disorders (such as anxiety or obsessive-compulsive disorders) may thus be smaller across all therapies but also in specific therapy approaches.

Psychotherapy differs from pharmacological treatments in terms of potential success after modification of the treatment plan: no HTE was found for antidepressant medication in previous studies (Plöderl \& Hengartner, 2019; Volkmann et al., 2020), so it is not surprising that switching antidepressants has no effect in non-responding patients (Bschor et al., 2018) while switching from antidepressants to psychotherapy following non-response is effective (Gloster et al., 2014). Indeed, the working mechanism behind pharmacological interventions may be the same in all patients, whereas this may not be the case for psychotherapy. However, research on the mechanisms of antidepressant medication is also not conclusive yet (Harmer et al., 2017). Another explanation of this difference in findings could be that our analysis focused on endpoint scores rather than changes from baseline. This was done mainly because the use of pre-post change scores was shown to be problematic, as they assume strong correlations between baseline and endpoint scores. This is violated in most psychotherapy trials (Cuijpers et al., 2017), which leads to reduced statistical power (Vickers, 2001) and, in many cases, a highly increased probability for false-positive findings (Bland \& Altman, 2011). Thus, it is generally recommended (Deeks et al., 2020; Vickers, 2001) to analyze endpoint scores as long as the relevant covariates are controlled for. To provide more conclusive evidence on the comparative HTE of psychotherapy and pharmacotherapy, individual patient data meta-analyses could be a way forward. These provide greater flexibility of analytic strategies, allow for systematic control of patient-level covariates like baseline severity, and enable researchers to conduct symptom-specific analyses of HTE. Certain sets of depressive symptoms may show greater HTE than others. Future studies should also consider studying HTE for methods designed to augment the effects of psychotherapy, as authors from this field argue that they may only work for certain subgroups of patients (Metcalf et al., 2020, p. 18).

Empirical studies testing the benefits of statistical treatment selection algorithms found effects that are in line with our HTE estimates. For example, after cross-validating a method that matches patients to either cognitive-behavioral therapy or interpersonal psychotherapy for depression, van Bronswijk et al. (2020) found that patients matched to their optimal treatment scored about 2 points or $9.1 \%$ lower on the BDI-II. Following a similar approach, Schwartz and colleagues (Schwartz et al., 2020) reported 14.2\% lower symptom severity on the Brief Symptom Inventory for the subgroup of patients that were predicted to benefit most from either CBT or PDT, which corresponds to a small effect (Cohen's $d=0.33$ ). Another study testing a treatment selection algorithm for PDT vs. CBT found a difference of 2.6 points on the HDRS (Cohen et al., 2020). For predictions of the advantage of either CBT or 
antidepressant medication, a difference of 3.6 points on the HDRS endpoint score was observed (DeRubeis et al., 2014). Finally, Deisenhofer et al. (2018) report an average difference of 2.7 points on the PHQ-9 when selecting either eye-movement desensitization and reprocessing (EMDR) or trauma-focused CBT. Some of these empirical findings could be upwardly biased due to a lack of cross-validation, although it can be assumed that none of them achieves perfect predictive accuracy for every case, which in turn reduces the benefit of the studied methods. In addition, most of these studies do not have the power to detect these small effects which could in the future be remedied by adhering to the HTE benchmarks provided in this study.

For clinical practice, two main conclusions can be drawn from our results. The demonstrated heterogeneity of treatment effects implies that session-by-session monitoring of outcomes in ongoing treatments is strongly advised (Lambert \& Harmon, 2018). This is also the case for interventions with a strong evidence base that are executed perfectly because HTE can be directly attributed to the treatment effect. We estimated the likelihood that a randomly selected patient will respond differently to a randomly selected treatment on the BDI-II to be $44 \%$. This indicates that a change of the treatment plan in the case of nonresponse could still lead to clinically meaningful effects. This shows that detecting nonresponding or deteriorating cases as early as possible is crucial for effective psychotherapy. Empirical evidence suggests that most of these cases are not recognized in time (Hatfield et al., 2009). Employing methods of session-by-session routine outcome monitoring could help clinicians to detect non-responding patients and adapt their treatment strategy (Lambert \& Harmon, 2018). Large meta-analyses demonstrate that these methods can increase treatment effects and decrease early termination rates in psychotherapy (de Jong et al., 2021; Lambert et al., 2018). Additionally, providing non-responding patients with a realistic estimate of treatment success after switching therapeutic approaches can be an important contribution to transparency in mental health.

Second, the results of this study stress the importance of prescriptive assessment. Since HTE can be assumed to be at least in part caused by patient-by-treatment interaction, close attention must be paid to prognostic and prescriptive patient characteristics. Well-validated, data-driven selection algorithms could help to decide which patient is most likely to profit from which intervention. As demonstrated by Hannan et al. (2005), statistically derived decision rules are more accurate than prognoses by clinicians for predicting treatment outcomes. An example of the successful application of a statistical treatment selection algorithm using routine care data was presented by Schwartz et al. (2021). Here, subgroups of patients likely to respond especially well to either cognitive-behavioral or psychodynamic psychotherapy were found, indicating that the use of treatment selection algorithms is not necessarily limited to highly controlled clinical studies. Another example is the Leeds Risk Index (LRI; Delgadillo et al., 2016), an algorithm developed to determine whether a given patient should be treated with guided self-help or face-to-face psychotherapy. The LRI's ability to improve outcomes was successfully tested under routine care conditions and it could be shown that it helps to improve outcomes for patient groups at risk for non-response (Delgadillo et al., 2020), which includes people with higher initial severity, disabilities, or unemployment. More recent studies on treatment prognosis and selection started to include easy-to-use calculators that enable practitioners to make more accurate predictions and guide the selection of interventions for individual patients. For example, Furukawa et al. (2021) used data from an individual patient data meta-analysis to predict response to various techniques and delivery methods of CBT. The calculator is freely available (https://esm.ispm.unibe.ch/shinies/cNMA_iCBT/) and can be used by providing only a small number of patient characteristics and allows the user to select the optimal number of intervention components to maximize treatment effects. Finally, advanced clinical support systems like the Trier Treatment Navigator (TTN; Lutz et al., 2019) provide prescriptive and 
adaptive treatment decisions for ongoing cases that are based on statistical models or other actuarial decision rules. In the case of the TTN, implementation in routine practice was shown to be feasible and an open-source version accessible free of charge is available (https://github.com/Psykli/Trier_Treatment_Navigator). It should be noted that implementation studies indicate that precise algorithms alone are not sufficient to ensure the success of a precision treatment decision support system, due to implementation issues making an algorithm less effective in clinical practice (Browning et al., 2021; Lutz et al., 2021). Additionally, statistical algorithms should be checked for systematic bias against certain groups that could lead to decreased predictive accuracy for underrepresented minority populations and could prevent them from accessing the care they need. Even though, for example, the aforementioned LRI increases the chances for disabled and unemployed persons to access high-intensity treatment, the sources of bias in data-driven algorithms need to be explored more (Obermeyer et al., 2019).

The widespread implementation of personalized treatments is influenced by the general availability of mental health treatment. Newly developed, empirically supported, and tailored internet interventions could help fill gaps in the availability of care (e.g., Păsărelu et al., 2017).

Regarding the training of future therapists, the results of this study suggest that a broader skillset of evidence-based therapeutic techniques is key to therapeutic success. Especially in combination with the aforementioned clinical support tools, this would enable future clinicians to flexibly adapt to patients' needs in the case of non-response. Current education and training in psychological interventions in most countries is based on different psychotherapy orientations or traditions such as psychodynamic therapy or CBT. Several authors argue that the identification with these specific schools of thought hinders the integration of different techniques and presents a barrier for scientifically informed development of personalized psychotherapy. The main reason for this is that theory-oriented training strongly limits the available techniques a single clinician can employ. Rief (2021) recently suggested moving from a tradition-based training approach to competence-based psychotherapy. Similarly, Gaines and Goldfried (2021) made a strong case for moving away from the traditional, theory-specific training model to a data-driven, patient-centered approach. The authors argue that training future psychotherapists in the application of transtheoretical mechanisms of change, enabling a more personalized approach to evidencebased psychotherapy. Reforming clinical training according to these proposals could also improve the dissemination of psychotherapy that is cognizant of HTE.

Some limitations of our study need to be mentioned. First, the trials included in our analysis used three different control conditions: passive control groups, where patients are randomized to a waiting list, or "control groups, where patients receive either "care as usual" or other types of control, including mostly interventions not designed to treat depression (like physical activity, meditation or relaxation exercises). This could have led to a biased estimation of VR and an increase in between-study-heterogeneity. As some treatment effects can still be expected in active control groups and "care as usual" (Blais et al., 2013), the inclusion of those studies could have led to a slightly higher variance in control groups and thus to lower estimates of HTE. Finally, the outcome measures used in the included studies were mostly measures of symptom severity. Although symptom reduction is considered an important goal in the treatment of depression (Chevance et al., 2020), other dimensions of improvement, such as health-related quality of life or functioning and adjustment, were not considered.

To conclude, our results demonstrate that patient-by-treatment interaction exists in psychotherapy for depression so that methods of personalizing interventions are likely to contribute to the improvement of treatment outcomes. Future studies on treatment selection should be powered to detect small effects resulting from personalization. Practitioners would 
do well to use established methods for early identification of non-response and evidencebased prognostic and prescriptive assessment and prediction methods. 


\section{References}

Ægisdóttir, S., White, M. J., Spengler, P. M., Maugherman, A. S., Anderson, L. A., Cook, R. S., Nichols, C. N., Lampropoulos, G. K., Walker, B. S., Cohen, G., \& Rush, J. D. (2006). The Meta-Analysis of Clinical Judgment Project: Fifty-six years of accumulated research on clinical versus statistical prediction. The Counseling Psychologist, 34(3), 341-382. https://doi.org/10/cbrmjf

Barron, F. (1953). Some test correlates of response to psychotherapy. Journal of Consulting Psychology, 17(4), 235-241. https://doi.org/10/b7rv3t

Beck, A. T., Steer, R. A., \& Brown, G. K. (1996). Beck Depression Inventory-II. Psychological Corporation.

Blais, M., Malone, J., Stein, M., Slavin-Mulford, J., O’Keefe, S., Renna, M., \& Sinclair, S. (2013). Treatment as Usual (TAU) for depression: A comparison of psychotherapy, pharmacotherapy, and combined treatment at a large academic medical center. Psychotherapy (Chicago, Ill.), 50, 110-118. https://doi.org/10.1037/a0031385

Bland, J. M., \& Altman, D. G. (2011). Comparisons against baseline within randomised groups are often used and can be highly misleading. Trials, 12(1), 264. https://doi.org/10.1186/17456215-12-264

Bobo, W. V., Angleró, G. C., Jenkins, G., Hall-Flavin, D. K., Weinshilboum, R., \& Biernacka, J. M. (2016). Validation of the 17-item Hamilton Depression Rating Scale definition of response for adults with major depressive disorder using equipercentile linking to Clinical Global Impression scale ratings: Analysis of Pharmacogenomic Research Network Antidepressant Medication Pharmacogenomic Study (PGRN-AMPS) data. Human Psychopharmacology: Clinical and Experimental, 31(3), 185-192. https://doi.org/10/f8mfz5

Browning, M., Bilderbeck, A. C., Dias, R., Dourish, C. T., Kingslake, J., Deckert, J., Goodwin, G. M., Gorwood, P., Guo, B., Harmer, C. J., Morriss, R., Reif, A., Ruhe, H. G., van Schaik, A., Simon, J., Sola, V. P., Veltman, D. J., Elices, M., Lever, A. G., ... Dawson, G. R. (2021). The clinical effectiveness of using a predictive algorithm to guide antidepressant treatment in primary care (PReDicT): An open-label, randomised controlled trial. Neuropsychopharmacology, 46(7), 1307-1314. https://doi.org/10/gjw3qw

Bschor, T., Kern, H., Henssler, J., \& Baethge, C. (2018). Switching the antidepressant after nonresponse in adults with Major Depression: A systematic literature search and metaanalysis. The Journal of Clinical Psychiatry, 79(1), 11-18. https://doi.org/10/gdvq9c

Bürkner, P.-C. (2017). Advanced Bayesian multilevel modeling with the R Package brms. ArXiv:1705.11123 [Stat]. http://arxiv.org/abs/1705.11123

Carmody, T. J., Rush, A. J., Bernstein, I., Warden, D., Brannan, S., Burnham, D., Woo, A., \& Trivedi, M. H. (2006). The Montgomery Åsberg and the Hamilton ratings of depression: A comparison of measures. European Neuropsychopharmacology, 16(8), 601-611. https://doi.org/10/b42dck

Carrozzino, D., Patierno, C., Fava, G. A., \& Guidi, J. (2020). The Hamilton Rating Scales for depression: A critical review of clinimetric properties of different versions. Psychotherapy and Psychosomatics, 89(3), 133-150. https://doi.org/10/ghjsqc

Chevance, A., Ravaud, P., Tomlinson, A., Le Berre, C., Teufer, B., Touboul, S., Fried, E. I., Gartlehner, G., Cipriani, A., \& Tran, V. T. (2020). Identifying outcomes for depression that matter to patients, informal caregivers, and health-care professionals: Qualitative content analysis of a large international online survey. The Lancet Psychiatry, 7(8), 692-702. https://doi.org/10/gg5v8j

Cipriani, A., Furukawa, T. A., Salanti, G., Chaimani, A., Atkinson, L. Z., Ogawa, Y., Leucht, S., Ruhe, H. G., Turner, E. H., Higgins, J. P. T., Egger, M., Takeshima, N., Hayasaka, Y., Imai, H., Shinohara, K., Tajika, A., Ioannidis, J. P. A., \& Geddes, J. R. (2018). Comparative efficacy and acceptability of 21 antidepressant drugs for the acute treatment of adults with major depressive disorder: A systematic review and network meta-analysis. The Lancet, 
391(10128), 1357-1366. https://doi.org/10/gcx763

Cohen, Z. D., \& DeRubeis, R. J. (2018). Treatment selection in depression. Annual Review of Clinical Psychology, 14(1), 209-236. https://doi.org/10/ghndkd

Cohen, Z. D., Kim, T. T., Van, H. L., Dekker, J. J. M., \& Driessen, E. (2020). A demonstration of a multi-method variable selection approach for treatment selection: Recommending cognitivebehavioral versus psychodynamic therapy for mild to moderate adult depression.

Psychotherapy Research, 30(2), 137-150. https://doi.org/10/gfs 8mj

Cooper, A. A., \& Conklin, L. R. (2015). Dropout from individual psychotherapy for major depression: A meta-analysis of randomized clinical trials. Clinical Psychology Review, 40, 57-65. https://doi.org/10.1016/j.cpr.2015.05.001

Cortés, J., González, J. A., Medina, M. N., Vogler, M., Vilaró, M., Elmore, M., Senn, S. J., Campbell, M., \& Cobo, E. (2019). Does evidence support the high expectations placed in precision medicine? A bibliographic review. F1000Research, 7. https://doi.org/10/gghqbp

Cuijpers, P., Karyotaki, E., Ciharova, M., Miguel, C., Noma, H., \& Furukawa, T. A. (2021). The effects of psychotherapies for depression on response, remission, reliable change, and deterioration: A meta-analysis. Acta Psychiatrica Scandinavica, 144(3), 288-299. https://doi.org/10.1111/acps.13335

Cuijpers, P., Karyotaki, E., Reijnders, M., \& Ebert, D. D. (2019). Was Eysenck right after all? A reassessment of the effects of psychotherapy for adult depression. Epidemiology and Psychiatric Sciences, 28(1), 21-30. https://doi.org/10.1017/s2045796018000057

Cuijpers, P., Karyotaki, E., Wit, L. de, \& Ebert, D. D. (2020). The effects of fifteen evidencesupported therapies for adult depression: A meta-analytic review. Psychotherapy Research, 30(3), 279-293. https://doi.org/10/ghjwkg

Cuijpers, P., Quero, S., Noma, H., Ciharova, M., Miguel, C., Karyotaki, E., Cipriani, A., Cristea, I. A., \& Furukawa, T. A. (2021). Psychotherapies for depression: A network meta-analysis covering efficacy, acceptability and long-term outcomes of all main treatment types. World Psychiatry, 20(2), 283-293. https://doi.org/10/gj34ss

Cuijpers, P., Weitz, E., Cristea, I. A., \& Twisk, J. (2017). Pre-post effect sizes should be avoided in meta-analyses. Epidemiology and Psychiatric Sciences, 26(4), 364-368. https://doi.org/10/gbphgd

de Jong, K., Conijn, J. M., Gallagher, R. A. V., Reshetnikova, A. S., Heij, M., \& Lutz, M. C. (2021). Using progress feedback to improve outcomes and reduce drop-out, treatment duration, and deterioration: A multilevel meta-analysis. Clinical Psychology Review, 85, 102002. https://doi.org/10/gjw3p8

Deeks, J. J., Higgins, J. P., \& Altman, D. G. (2020). Chapter 10: Analysing data and undertaking meta-analyses. In J. P. Higgins, J. Chandler, M. Cumpston, T. Li, M. Page, \& V. Welch (Eds.), Cochrane handbook for systematic reviews of interventions, version 6.1.

Deisenhofer, A.-K., Delgadillo, J., Rubel, J. A., Böhnke, J. R., Zimmermann, D., Schwartz, B., \& Lutz, W. (2018). Individual treatment selection for patients with posttraumatic stress disorder. Depression and Anxiety, 35(6), 541-550. https://doi.org/10/ggxdg3

Delgadillo, J., Appleby, S., Booth, S., Burnett, G., Carey, A., Edmeade, L., Green, S., Griffin, P., Johnson, E., Jones, R., Parker, P., Reeves-McLaren, L., \& Lutz, W. (2020). The Leeds Risk Index: Field-test of a stratified psychological treatment selection algorithm. Psychotherapy and Psychosomatics, 89(3), 189-190. https://doi.org/10/gjw32m

Delgadillo, J., Moreea, O., \& Lutz, W. (2016). Different people respond differently to therapy: A demonstration using patient profiling and risk stratification. Behaviour Research and Therapy, 79, 15-22. https://doi.org/10/f8h624

DeRubeis, R. J., Cohen, Z. D., Forand, N. R., Fournier, J. C., Gelfand, L. A., \& Lorenzo-Luaces, L. (2014). The Personalized Advantage Index: Translating research on prediction into individualized treatment recommendations. A demonstration. PLOS ONE, 9(1), e83875. https://doi.org/10.1371/journal.pone.0083875 
Enck, P., \& Zipfel, S. (2019). Placebo effects in psychotherapy: A Framework. Frontiers in Psychiatry, 10. https://doi.org/10.3389/fpsyt.2019.00456

Furukawa, T. A., Shinohara, K., Sahker, E., Karyotaki, E., Miguel, C., Ciharova, M., Bockting, C. L. H., Breedvelt, J. J. F., Tajika, A., Imai, H., Ostinelli, E. G., Sakata, M., Toyomoto, R., Kishimoto, S., Ito, M., Furukawa, Y., Cipriani, A., Hollon, S. D., \& Cuijpers, P. (2021). Initial treatment choices to achieve sustained response in major depression: A systematic review and network meta-analysis. World Psychiatry, 20(3), 387-396. https://doi.org/10/gms $44 \mathrm{z}$

Furukawa, T. A., Suganuma, A., Ostinelli, E. G., Andersson, G., Beevers, C. G., Shumake, J., Berger, T., Boele, F. W., Buntrock, C., Carlbring, P., Choi, I., Christensen, H., Mackinnon, A., Dahne, J., Huibers, M. J. H., Ebert, D. D., Farrer, L., Forand, N. R., Strunk, D. R., ... Cuijpers, P. (2021). Dismantling, optimising, and personalising internet cognitive behavioural therapy for depression: A systematic review and component network metaanalysis using individual participant data. The Lancet Psychiatry, 8(6), 500-511. https://doi.org/10/gjvntk

Gaines, A. N., \& Goldfried, M. R. (2021). Consensus in psychotherapy: Are we there yet? Clinical Psychology: Science and Practice. https://doi.org/10/gmwx87

Goldberg, D. (2011). The heterogeneity of "major depression." World Psychiatry, 10(3), 226-228. https://doi.org/10/gmvvzp

Hamilton, M. (1960). A rating scale for depression. Journal of Neurology, Neurosurgery, and Psychiatry, 23, 56-62. https://doi.org/10.1136/jnnp.23.1.56

Hannan, C., Lambert, M. J., Harmon, C., Nielsen, S. L., Smart, D. W., Shimokawa, K., \& Sutton, S. W. (2005). A lab test and algorithms for identifying clients at risk for treatment failure. Journal of Clinical Psychology, 61(2), 155-163. https://doi.org/10.1002/jclp.20108

Harmer, C. J., Duman, R. S., \& Cowen, P. J. (2017). How do antidepressants work? New perspectives for refining future treatment approaches. The Lancet. Psychiatry, 4(5), 409-418. https://doi.org/10.1016/s2215-0366(17)30015-9

Hatfield, D., McCullough, L., Frantz, S. H. B., \& Krieger, K. (2009). Do we know when our clients get worse? An investigation of therapists' ability to detect negative client change. Clinical Psychology \& Psychotherapy, 17, 15-32. https://doi.org/10.1002/cpp.656

Hiroe, T., Kojima, M., Yamamoto, I., Nojima, S., Kinoshita, Y., Hashimoto, N., Watanabe, N., Maeda, T., \& Furukawa, T. A. (2005). Gradations of clinical severity and sensitivity to change assessed with the Beck Depression Inventory-II in Japanese patients with depression. Psychiatry Research, 135(3), 229-235. https://doi.org/10/cmznt7

Kessler, R. C., \& Luedtke, A. (2021). Pragmatic precision psychiatry-A new direction for optimizing treatment selection. JAMA Psychiatry, 78(12), 1384. https://doi.org/10/gnpk28

Kriston, L., von Wolff, A., Westphal, A., Hölzel, L. P., \& Härter, M. (2014). Efficacy and acceptability of acute treatments for persistent depressive disorder: A network meta-analysis. Depression and Anxiety, 31(8), 621-630. https://doi.org/10/f6dfrf

Kroenke, K., \& Spitzer, R. L. (2002). The PHQ-9: A new depression diagnostic and severity measure. Psychiatric Annals, 32(9), 509-515. https://doi.org/10/gf6zrm

Lambert, M. J., \& Harmon, K. L. (2018). The merits of implementing routine outcome monitoring in clinical practice. Clinical Psychology: Science and Practice, 25(4). https://doi.org/10/gftk6b

Lambert, M. J., Whipple, J. L., \& Kleinstäuber, M. (2018). Collecting and delivering progress feedback: A meta-analysis of routine outcome monitoring. Psychotherapy, 55(4), 520-537. https://doi.org/10/gfgm $4 \mathrm{k}$

Leucht, S., Fennema, H., Engel, R. R., Kaspers-Janssen, M., Lepping, P., \& Szegedi, A. (2017). What does the MADRS mean? Equipercentile linking with the CGI using a company database of mirtazapine studies. Journal of Affective Disorders, 210, 287-293. https://doi.org/10/f9xjgt

Levis, B., Benedetti, A., \& Thombs, B. D. (2019). Accuracy of Patient Health Questionnaire-9 
(PHQ-9) for screening to detect major depression: Individual participant data meta-analysis. $B M J, 11476$. https://doi.org/10/ggskgf

Lorenzo-Luaces, L., Peipert, A., De Jesús Romero, R., Rutter, L. A., \& Rodriguez-Quintana, N. (2020). Personalized medicine and cognitive behavioral therapies for depression: Small effects, big problems, and bigger data. International Journal of Cognitive Therapy. https://doi.org/10/gjw3vr

Löwe, B., Unützer, J., Callahan, C. M., Perkins, A. J., \& Kroenke, K. (2004). Monitoring depression treatment outcomes with the Patient Health Questionnaire-9: Medical Care, 42(12), 11941201. https://doi.org/10/ds6ptr

Luedtke, A., \& Kessler, R. C. (2021). New directions in research on heterogeneity of treatment effects for Major Depression. JAMA Psychiatry, 78(5), 478. https://doi.org/10/gkgk36

Lutz, W., Deisenhofer, A.-K., Rubel, J., Bennemann, B., Giesemann, J., Poster, K., \& Schwartz, B. (2021). Prospective evaluation of a clinical decision support system in psychological therapy. Journal of Consulting and Clinical Psychology. https://doi.org/10/gmvvx 7

Lutz, W., Rubel, J. A., Schwartz, B., Schilling, V., \& Deisenhofer, A.-K. (2019). Towards integrating personalized feedback research into clinical practice: Development of the Trier Treatment Navigator (TTN). Behaviour Research and Therapy, 120, 103438. https://doi.org/10/gjjwtk

Metcalf, O., Stone, C., Hinton, M., O’Donnell, M., Hopwood, M., McFarlane, A., Forbes, D., Kartal, D., Watson, L., Freijah, I., \& Varker, T. (2020). Treatment augmentation for posttraumatic stress disorder: A systematic review. Clinical Psychology: Science and Practice, 27(1). https://doi.org/10/ggf4f6

Moeyaert, M., Ugille, M., Natasha Beretvas, S., Ferron, J., Bunuan, R., \& Van den Noortgate, W. (2017). Methods for dealing with multiple outcomes in meta-analysis: A comparison between averaging effect sizes, robust variance estimation and multilevel meta-analysis. International Journal of Social Research Methodology, 20(6), 559-572. https://doi.org/10/ggwwhs

Montgomery, S. A., \& Åsberg, M. (1979). A New Depression Scale Designed to be Sensitive to Change. British Journal of Psychiatry, 134(4), 382-389. https://doi.org/10/fgz9gw

Nakagawa, S., Poulin, R., Mengersen, K., Reinhold, K., Engqvist, L., Lagisz, M., \& Senior, A. M. (2015). Meta-analysis of variation: Ecological and evolutionary applications and beyond. Methods in Ecology and Evolution, 6(2), 143-152. https://doi.org/10/f627v9

Obermeyer, Z., Powers, B., Vogeli, C., \& Mullainathan, S. (2019). Dissecting racial bias in an algorithm used to manage the health of populations. Science, 366(6464), 447-453. https://doi.org/10.1126/science.aax 2342

Paul, G. L. (1967). Strategy of outcome research in psychotherapy. Journal of Consulting Psychology, 31(2), 109-118. https://doi.org/10.1037/h0024436

Plöderl, M., \& Hengartner, M. P. (2019). What are the chances for personalised treatment with antidepressants? Detection of patient-by-treatment interaction with a variance ratio metaanalysis. BMJ Open, $9(12)$, e034816. https://doi.org/10/gjw3x3

Price, M., Legrand, A. C., Brier, Z. M. F., \& Hébert-Dufresne, L. (2019). The symptoms at the center: Examining the comorbidity of posttraumatic stress disorder, generalized anxiety disorder, and depression with network analysis. Journal of Psychiatric Research, 109, 52-58. https://doi.org/10/ghn6zc

$\mathrm{R}$ Core Team. (2020). $R$ : A language and environment for statistical computing. $\mathrm{R}$ Foundation for Statistical Computing. https://www.R-project.org/

Rief, W. (2021). Moving from tradition-based to competence-based psychotherapy. Evidence-Based Mental Health, 24(3), 115-120. https://doi.org/10.1136/ebmental-2020-300219

Schwartz, B., Cohen, Z. D., Rubel, J. A., Zimmermann, D., Wittmann, W. W., \& Lutz, W. (2020). Personalized treatment selection in routine care: Integrating machine learning and statistical algorithms to recommend cognitive behavioral or psychodynamic therapy. Psychotherapy Research, 0(0), 1-19. https://doi.org/10/ghbwb2 
Simon, G. E., \& Perlis, R. H. (2010). Personalized medicine for depression: Can we match patients with treatments? American Journal of Psychiatry, 167(12), 1445-1455. https://doi.org/10/dgb8kd

Stegenga, B. T., Kamphuis, M. H., King, M., Nazareth, I., \& Geerlings, M. I. (2012). The natural course and outcome of major depressive disorder in primary care: The PREDICT-NL study. Social Psychiatry and Psychiatric Epidemiology, 47(1), 87-95. https://doi.org/10/dzksq2

Steinert, C., Hofmann, M., Kruse, J., \& Leichsenring, F. (2014). Relapse rates after psychotherapy for depression - stable long-term effects? A meta-analysis. Journal of Affective Disorders, 168, 107-118. https://doi.org/10/f6f6m2

Swift, J. K., \& Greenberg, R. P. (2012). Premature discontinuation in adult psychotherapy: A metaanalysis. Journal of Consulting and Clinical Psychology, 80(4), 547-559. https://doi.org/10.1037/a0028226

Van Bronswijk, S. C., Bruijniks, S. J. E., Lorenzo-Luaces, L., Derubeis, R. J., Lemmens, L. H. J. M., Peeters, F. P. M. L., \& Huibers, Marcus. J. H. (2020). Cross-trial prediction in psychotherapy: External validation of the Personalized Advantage Index using machine learning in two Dutch randomized trials comparing CBT versus IPT for depression. Psychotherapy Research, 1-14. https://doi.org/10/gh3kdw

Vickers, A. J. (2001). The use of percentage change from baseline as an outcome in a controlled trial is statistically inefficient: A simulation study. BMC Medical Research Methodology, 1(1), 6. https://doi.org/10/fr5djv

Volkmann, A. (2020). On the relationship between treatment effect heterogeneity and the variability ratio effect size statistic. ArXiv:2006.11848 [Stat]. http://arxiv.org/abs/2006.11848

Volkmann, C., Volkmann, A., \& Müller, C. A. (2020). On the treatment effect heterogeneity of antidepressants in major depression: A Bayesian meta-analysis and simulation study. PLOS ONE, 15(11), e0241497. https://doi.org/10/gjw3wh

Wampold, B. E. (2015). How important are the common factors in psychotherapy? An update. World Psychiatry, 14(3), 270-277. https://doi.org/10/f7x62f

Wang, Y.-P., \& Gorenstein, C. (2013). Psychometric properties of the Beck Depression Inventory-II: A comprehensive review. Brazilian Journal of Psychiatry, 35, 416-431. https://doi.org/10.1590/1516-4446-2012-1048

Winkelbeiner, S., Leucht, S., Kane, J. M., \& Homan, P. (2019). Evaluation of differences in individual treatment response in schizophrenia spectrum disorders: A meta-analysis. JAMA Psychiatry, 76(10), 1063. https://doi.org/10/ghgsk9 